\title{
Competitive Algorithms for Bulk Allocation under Uncertainty: The Caterer's Problem
}

\author{
Arjun Kodialam \\ Marlboro High School \\ Marlboro, NJ
}

\begin{abstract}
We consider the problem of allocating resources to meet an uncertain demand. There are well studied approaches to solve this problem when distributional information is known about the demand. We consider this resource allocation problem when we lack information about the statistics of the demand. These types of resource allocation problems, where there is only minimal information available about the demand, arises naturally in many instances. The motivation for studying this problem is the allocation of resources (testing kits, masks) for pandemic control. While resources are being allocated, there is only minimal information known about the demand size. Decision makers have to solve some version of the Caterer's problem when making advance reservation of resources (processing, storage) in the cloud for new applications. We develop deterministic and randomized competitive algorithms for the Caterer's problem. Unlike the closely related online Ski-rental problem, the Caterer's problem does not satisfy the principle of equality and this makes the Caterer's problem more challenging. We prove the optimality of the randomized algorithms by using Yao's Lemma and developing matching lower bounds for the problem.
\end{abstract}

\section{INTRODUCTION}

In many realistic scenarios, resource allocation decisions are made under uncertainty. When allocation decisions are made, a decision maker may only have access to limited, possibly probabilistic information about potential resource demands in the future. Nevertheless, the decision maker seeks to minimize cost or maximize profit using the available information.

Several paradigmatic resource allocation frameworks can be captured by simple examples. In a single stage resource allocation problem, the decision maker only makes a single resource allocation decision under uncertainty. A classical example of a single stage resource allocation problem is the newsvendor problem. [1]

In the newsvendor problem [2], a newsvendor decides the number of newspapers to be ordered on a given day. The quantity of newspapers that will be demanded is unknown, and any leftover newspapers are discarded. Each newspaper sold generates a profit, and each newspaper ordered incurs a cost and the objective of the newsvendor is to determine the number of newspapers to order to maximize the expected profit. In the case where the distribution of the number of newspapers that will be demanded is known, this problem is a crucial building block of stochastic inventory theory and is studied extensively in the management science literature [3,
4].

There are, however, many instances where the demand distribution is not known ahead of time. In this case, it is not possible to minimize the expected cost or maximize the expected profit. When no information is available about distribution of the demand, alternative formulations are needed. One approach [5] is a decison theoretic approach based on a hybrid of Hurwicz and Bayes decision rules that takes into account the decision maker's attitude towards risk. Another approach developed in [6] uses a possibility distribution instead of a probability distribution to characterize the demand. In this paper, we develop an alternative approach to mitigate worst-case outcomes [7] . In contrast to single stage resource allocation, a multi stage resource allocation problem involves the decision maker performing multiple resource allocations. The ski rental problem [8] is a quintessential example of this paradigm.

In the ski-rental problem, a skier decides at the beginning of each day whether to rent skis for that day for 1 dollar, or to buy skis for $b$ dollars. However, the skier does not know how many days of skiing are left in the season - if she skis for less than $b$ days the optimal strategy is to rent the skis on each day, but if she skis for more than $b$ days it is optimal to buy the skis immediately. Since the number of skiing days is not known ahead of time the online approach is to develop a strategy that performs well compared to the optimal strategy in the worst-case situation. Fundamentally, the ski rental problem allows us to develop a framework in which allocation decisions are made incrementally without any information about the future.

In this paper, we develop an algorithm for a single allocation decision made with limited knowledge of the future. The motivation for the problem is deciding the quantity of safety and testing equipment to pre-stock in preparation for health crises. The demand for such equipment, or even the distribution of the demand for such equipment, may be functionally unknown especially if the health issue in question is poorly understood. If the allocation is insufficient, then additional supplies must be obtained at the last minute. Doing so may incur a fixed cost (independent of the quantity ordered), as well as variable costs per unit of additional resources. Notably, the per-unit cost for resources at a time of need may be significantly different from the cost to stockpile a single unit.

This decision problem is a single stage optimization problem like the newsvendor problem, and the decision maker does not have any information about the demand distribution. However, the unknown demand resembles the unknown number of ski days in the ski-rental problem. As in 
previous works, we motivate this novel single stage decision problem under uncertainty using a stylized framework called the Caterer's Problem.

\subsection{Caterer's Problem.}

A caterer enters into a contract to serve all people attending an event. The number of people attending the event is not known in advance, but a lower bound $\ell \geq 1$ and an upper bound $u \leq \infty$ on the number of guests may be known. The caterer has to decide how many plates to prepare without advance knowledge of the number of guests.

We scale costs such that the cost to the caterer to prepare one plate is 1 dollar. If the number of plates prepared is greater than the number of guests, excess plates are wasted. However, if the initial number of plates prepared is less than the number of guests, then the caterer must prepare additional plates at a cost of $\alpha$ dollars per plate, and will also incur a fixed charge of $b$ dollars to get these additional supplies to the event. The caterer's objective is to choose is the number of plates to prepare in advance such that the total cost of fulfilling the contract is minimized.

Remarks.

(1) It is easy to include a fixed cost to the initial allocation. This cost will be incurred by all algorithms. The performance bounds derived in this paper will provide an upper bound on the performance if there is a fixed cost for the initial allocation.

(2) The assumption that the initial allocation is made at unit cost is without loss of generality. If the initial cost per unit is $c$ dollars, then we can scale down the fixed charge $b$ and the cost of providing additional resources $\alpha$ by $c$ (i.e., make these costs $b / c$ and $\alpha / c$ respectively) and re-formulate the problem with unit cost.

The Caterer's problem can be used to model several single stage stochastic allocation problems.

- An example of $\alpha=0$ is a long term leasing problem. In this problem, a customer wants to enter into a multiperiod lease for some item. The customer knows that he is going to need the item for between $\ell$ and $u$ time periods. The cost of leasing the item per period is one dollar and the cost of buying the item is $b$ dollars. Unlike the ski-rental problem, the customer cannot extend the lease at the end of each time period but has to commit to a multi-time period lease. A natural question to ask is how long should the initial leasing period be before the customer buys the item (if necessary).

- The $\alpha=0$ case also models the problem of a contract with a non completion penalty of $b$. These kinds of contracts with contract violation penalties are commonly used to avoid litigation if a contract is not completed.

- With $\alpha>1$, then the caterers problem can be used to model the problem of ordering test kits where the cost of providing one unit of additional resource can be greater that the initial allocation cost due to increased market demand. As in the Caterer's Problem, $b$ can be viewed as the fixed cost of getting the additional resources.

- With the increasing use of the cloud to provide computing infrastructures, companies enter into advanced reservation contracts with cloud service providers for processing and storage resources. The cost of providing resources are cheaper if they are made in advance. Companies have to estimate future needs and make the reservations but the actual need may be different from the estimated needs. If additional resources have to be purchased in real time, then the cost of these resources will be typically be higher.

Like the newsvendor problem, the caterer's problem is a one stage decision problem. Since the distribution of the number of guests is not known, we use the competitive ratio to measure the efficiency of the solution to the Caterer's problem.

\subsection{Competitive Ratio}

The caterer would ideally like to meet the demand at minimum total cost. Since the demand or any probabilistic information about the demand is not known ahead of time, the caterer has to develop a strategy that gets close to the minimum cost solution for the worst case demand. This idea of computing solutions to problems where there is uncertainty in the inputs has been used extensively in the computer science literature [7]. The most common approach to handling uncertainty in the input is the idea of competitive ratio [8]. Let $y$ denote the unknown input to the algorithm. In our case $y$ is the unknown demand. Let $O P T(y)$ denote the solution to the optimization problem when the value of $y$ is known. Let $A L G(y)$ denote the solution provided by the algorithm when the input is $y$. The competitive ratio $\lambda_{A L G}$ of the algorithm $A L G$ is defined as

$$
\lambda_{A L G}=\max _{y} \frac{A L G(y)}{O P T(y)} .
$$

In other words, the competitive ratio of $A L G$ is the worst case ratio of the solution provided by the algorithm to the optimal solution over all the inputs. We would like the competitive ratio of the algorithm to be as small as possible. Note that $\lambda_{A L G} \geq 1$ since $O P T(y)$ is solution when the value of $y$ is known and it is not possible to do better. The challenge is to develop an algorithm $A L G$ that comes close to $O P T$ for all possible values of $y$.

\subsection{Deterministic and Randomized Competitive Algorithms}

If the algorithm $A L G$ does not use any randomness and finds a solution independent of the input then it is termed a deterministic algorithm. For some problems, including the Caterer's problem, it is possible to do better if the algorithm uses a random initial allocation. If randomization is used, then the value of the solution provided by $A L G$ will be a random variable. In this case, we use $E[A L G(y)]$ to denote the expected value of the solution provided by the randomized 
algorithm $A L G$ when the input is $y$. Note that the randomized algorithm cannot use any information about $y$ when performing randomization. The performance of a randomized algorithm is the expected competitive ratio that is defined as

$$
\lambda_{A L G}=\max _{y} \frac{E[A L G(y)]}{O P T(y)} .
$$

We develop a randomized algorithm and show that its competitive ratio is at most that of the optimal deterministic algorithm.

\subsection{Solution Technique}

The techniques used for the Ski-rental problem cannot be extended directly to the Caterer's problem. This is due to the fact that the Ski-rental problem has the property of principle of equality [9]. This property ensures that the competitive ratio for the problem is constant across the entire range of the unknown parameter. This is exploited either explicitly or implicitly in the analysis of Ski-rental and similar online problems. In fact [9] has unified these techniques to derive solutions to a wide range of online algorithms. However this technique cannot be used for the Caterer's problem. For the Caterer's problem, the competitive ratio is at the highest value upto some value of the parameter and then decreases. A critical component of the analysis it to find the threshold value of the parameter until which the competitive ratio remains at its worst case value. If the upper bound $u$ on the demand is low, then the principle of equality will hold and we can use a modified version of the analysis of the Ski-rental problem in [9] to derive a competitive algorithm. For the general Caterer's problem part of the analysis is to find the threshold until which the competitive ratio remains at its worst case value.

\section{PROBLEM DEFINITION}

We now formally define the Caterer's problem. Assume that a caterer has agreed to cater to all the people attending an event. The only information known about the number of people (demand) $y$ attending the event is that $\ell \leq y \leq u$ where $\ell \geq 1$ and $u \leq \infty$. The caterer has to serve one plate to each guest. Without loss of generality, the cost of each plate is one dollar. The caterer has to decide the number of plates (initial allocation) $x$ that he is going to prepare in advance. If the demand $y \leq x$, then no additional cost is incurred and the total cost is $x$. The excess resource $x-y$ is not utilized. If the demand $y>x$, then the caterer has to augment the number of plates to meet the demand. Augmenting resources incurs a fixed cost of $b$ whenever there is any augmentation and a per unit cot of $\alpha$ dollars. Augmenting $y-x$ plates incurs a augmenting cost of $b+\alpha(y-x)$ dollars and a total cost of $x+b+\alpha(y-x)$. Therefore, when an initial assignment $x$ is made when the demand is $y$, the total cost $T C(x, y)$ is

$$
T C(x, y)= \begin{cases}x+b+\alpha(y-x) & \text { if } x<y \\ x & \text { if } x \geq y\end{cases}
$$

If the demand $y$ is known ahead of time, then the optimal cost of meeting the demand $O P T(y)$ is

$$
O P T(y)=\min \{y, b+\alpha y\} .
$$

The first term is to assign $y$ initially, incurring a cost of $y$. The second term is the cost incurred when the initial allocation $x=0$ and the augmentation cost is $b+\alpha y$. The optimum cost is the minimum of these two options. Note that the second option can be optimal for $y \geq \frac{b}{1-\alpha}$ if $\alpha<1$. The competitive ratio $\lambda$ is defined as the worst case ratio of the cost incurred by the algorithm to the optimal cost.

$$
\lambda_{d}=\min _{x} \max _{\ell \leq y \leq u} \frac{T C(x, y)}{O P T(y)} .
$$

If a randomized algorithm is used, then the initial allocation is a random variable $X$ with density function $p(x)$. The objective of the randomized algorithm is to determine $p(x)$ such that

$$
\lambda_{r}=\min _{p(x)} \max _{\ell \leq y \leq u} \frac{E_{x}[T C(X, y)]}{O P T(y)}
$$

where $E_{x}[$ is the expectation with respect to density function $p(x)$. We assume that the demand $y$ is picked by an adversary who has knowledge of the choice made by the optimizer. In the case where the optimizer uses a randomized strategy, we assume an oblivious adversary [7] that knows the strategy but does not know the exact outcome of the randomization process. We now outline a deterministic algorithm to obtain the best competitive ratio for the Caterer's problem.

\section{DETERMINISTIC COMPETITIVE ALGORITHM}

In a deterministic strategy, the caterer develops an approach that makes the initial allocation $x$ a function of the known problem parameters: the lower bound $\ell$, the upper bound $u$, the fixed cost $b$ and the per unit augmenting cost $\alpha$. The general approach for determining the worst case demand $y$ that maximizes the competitive ratio is to assume that $y$ is picked by an adversary that knows the value of $x$. In order to keep the derivation of the competitive ratio simple, we first show a simple result that bounds the optimal deterministic allocation $x^{*}$ for the case when $\alpha<1$. This result helps us unify the analysis for the cases $\alpha<1$ and $\alpha \geq 1$ in the rest of the paper.

LEMMA 3.1. If $\alpha<1$, then the initial assignment $x^{*}$ that minimizes the competitive ratio satisfies $x^{*} \leq \frac{b}{1-\alpha}$.

PROOF. We prove this result by contradiction. Assume that the minimum competitive ratio is attained by an initial assignment $x^{*}>\frac{b}{1-\alpha}$. If the adversary picks a demand $y<x^{*}$, then the worst case competitive ratio is attained when $y=\ell$. In this case the competitive ratio is $\frac{x^{*}}{\ell}$ which decreases when $x^{*}$ decreases. If the adversary picks $y>x^{*}$, then the cost for the caterer is $x^{*}+b+\alpha\left(y-x^{*}\right)$ and the optimum cost is $b+\alpha y$ (since $y>x^{*}>\frac{b}{1-\alpha}$ ). Therefore the competitive ratio is

$$
\frac{x^{*}+b+\alpha\left(y-x^{*}\right)}{b+\alpha y}=1+\frac{(1-\alpha) x^{*}}{b+\alpha y} \text {. }
$$


Since $\alpha<1$, this is also an increasing function of $x^{*}$. Therefore, the competitive ratio can be decreased by decreasing $x^{*}$. This contradicts the assumption that $x^{*}$ attains the minimum competitive ratio.

Since the adversary knows that $x^{*} \leq \frac{b}{1-\alpha}$, he will also set $y \leq \frac{b}{1-\alpha}$. In this case $O P T(y)=y$. When $\alpha>1$ the the optimal cost $O P T(y)=y$. Therefore, when computing the competitive ratio, we can assume that the optimal cost is $y$ for all values of $\alpha$. We can now outline the performance of a deterministic competitive algorithm for our problem.

THEOREM 3.2. There is a deterministic competitive algorithm with a competitive ratio of

$$
\lambda_{d}=\max \left\{\frac{1}{2}\left[1+\sqrt{1+\frac{4 b}{\ell}}\right], \alpha\right\}
$$

Proof. Assume that the caterer picks an initial assignment of $x^{*}$. If the adversary picks a demand $y \leq x^{*}$, then the worst case competitive ratio is attained when $y=\ell$ and the competitive ratio is $\frac{x^{*}}{\ell}$. We call this the left competitive ratio. If the adversary picks some $y>x^{*}$ then the worst case competitive ratio is

$$
\frac{x^{*}+b+\alpha\left(y-x^{*}\right)}{y}=\alpha+\frac{b+(1-\alpha) x^{*}}{y} .
$$

By Lemma 3.1 we know that the optimal cost is $y$. The adversary wants to make this expression as large as possible. The maximum value is called the the right competitive ratio. There are two cases to consider to compute the right competitive ratio.

- If $b+(1-\alpha) x^{*} \geq 0$, then the adversary sets $y=x^{*}+\epsilon$ and the right competitive ratio is

$$
\lim _{\epsilon \rightarrow 0} \frac{x^{*}+b}{x^{*}+\epsilon}=1+\frac{b}{x^{*}}
$$

The caterer determines the initial assignment $x^{*}$ that makes the left and right competitive ratios equal.

$$
\frac{x^{*}}{\ell}=1+\frac{b}{x^{*}} .
$$

Solving for $x^{*}$, we get

$$
x^{*}=\frac{1}{2}\left[\ell+\sqrt{\ell^{2}+4 b \ell}\right]
$$

and the optimal competitive ratio $\frac{x^{*}}{\ell}$ is

$$
\lambda_{d}^{1}=\frac{1}{2}\left[1+\sqrt{1+4\left(\frac{b}{\ell}\right)}\right]
$$

- If $b+(1-\alpha) x^{*}<0$, then the adversary can set $y=\infty$ to make the right competitive ratio $\alpha$. In this case, the overall competitive ratio of the algorithm is $\alpha$.

- If $b+(1-\alpha) x^{*}<0$ then
The critical value of $\alpha$ (denoted by $\alpha_{c}$ ) above which the competitive ratio is $\alpha$ is

$$
\alpha_{c}=\frac{1}{2}\left[1+\sqrt{1+4\left(\frac{b}{\ell}\right)}\right]
$$

After some algebra, this condition can also be written as

$$
b+\alpha_{c} \ell-\alpha_{c}^{2} \ell=0
$$

Therefore,

$$
\lambda_{d}= \begin{cases}\frac{1}{2}\left[1+\sqrt{1+4\left(\frac{b}{\ell}\right)}\right] & \text { if } b+\alpha \ell-\alpha^{2} \ell>0 \\ \alpha & \text { if } b+\alpha \ell-\alpha^{2} \ell \leq 0\end{cases}
$$

A natural question to ask is whether the caterer can improve its performance by randomization. In the next section, we show that the expected competitive ratio of a randomized competitive algorithm is at least as good as the deterministic algorithm.

\section{RANDOMIZED COMPETITIVE ALGORITHM}

We now see how to exploit randomness to improve the expected competitive ratio. In a randomized algorithm, the initial allocation $X$ is a random variable. Therefore the competitive ratio is also a random variable. We choose the probability density function of $X$ to minimize the expected competitive ratio. The algorithms that we derive in this section are optimal randomized algorithm. A randomized competitive algorithm is termed optimal, if we can show that it is not possible to derive any randomized algorithm that has lower competitive ratio. This is shown by deriving a lower bound on the achievable competitive ratio using Yao's principle [10]. The functional form of the probability density function that minimizes the competitive ratio is different for the case when unit incremental cost $\alpha=0$ and when the unit incremental $\operatorname{cost} \alpha>0$. Therefore, we treat these two cases separately. It is possible to use limiting arguments to derive the optimal pdf for the case where $\alpha=0$ from the general case but we outline a direct derivation. We follow the following steps in the derivation of the randomized competitive algorithm.

- We ignore the upper bound $u$ (equivalent to setting $u=\infty$ ) and derive a randomized competitive algorithm for the $\alpha=0$ case in Section 4.1 and for $\alpha>0$ in Section 4.2 .

- In Section 5, we show how to modify the procedure where the upper bound $u$ is smaller than the threshold derived in Section 4.2.

- The matching lower bounds are derived in Section 5 that show that the derived randomized competitive algorithms are optimal.

Since a deterministic competitive algorithm is a special case of a randomized competitive algorithm (where all the weight is placed at one allocation), the optimal randomized competitive algorithm always does at least as well as a deterministic algorithm. 


\subsection{Randomized Algorithm for $\alpha=0$ for Large} $u$

In this section we assume that the unit cost for augmentation is zero and the upper bound $u=\infty$. Assume that the caterer picks the initial allocation randomly. The adversary who picks the actual demand $y$ knows that the caterer is randomizing as well as the pdf of the initial allocation. The adversary, however, does not know the actual initial allocation $X$ (which is a random variable) drawn by the caterer. Let $p(x)$ denote the probability density function of $X$. Let $\mathbb{E}[X]$ denote the expected value of $X$. The expected cost $\mathbb{E}\left[C^{\prime}(y)\right]$ when $x$ is picked using the probability density function $p(x)$ and the actual demand is $y$ is given by

$$
\begin{aligned}
\mathbb{E}\left[C^{\prime}(y)\right] & =\int_{\ell}^{y}(x+b) p(x) d x+\int_{y}^{\infty} x p(x) d x \\
& =\mathbb{E}[X]+b \int_{\ell}^{y} p(x) d x
\end{aligned}
$$

If the demand is $y$, then the optimal cost is $y$. If $\lambda_{r}$ is the expected competitive ratio then,

$$
\mathbb{E}[X]+b \int_{\ell}^{y} p(x) d x=\lambda_{r} y .
$$

Differentiating this with respect to $y$, then

$$
b p(y)=\lambda_{r} .
$$

Therefore $p(x)$ is a constant. Assume that $p(x)$ is a uniform distribution between $\ell$ and (to be determined) upper bound $w$. This is where the derivation of the optimal pdf varies from the optimal pdf for the standard ski-rental problem. In the ski-rental problem, it is easy to show [7]) that the pdf is upper bounded by the fixed cost $b$ and the optimal expected competitive ratio is constant in the interval $[1, b]$. This has also been referred to as the principle of equality ([9] and is crucial for deriving the optimal pdf. We not not have this property for the caterer's problem. If we assume that the competitive ratio is constant in the interval $[\ell, b]$ it leads to a sub-optimal pdf. Therefore, determining the upper bound is an important component of deriving the optimal pdf. In Section 6, we show that the value of $w$ is also part of the pdf to prove the optimality of the derived competitive ratio. We define the pdf $p(x)$ as

$$
p(x)= \begin{cases}\frac{1}{w-\ell} & \text { if } \ell \leq x \leq w \\ 0 & \text { Otherwise }\end{cases}
$$

Therefore the expected cost $\mathbb{E}\left[C^{\prime}(y)\right]$ is

$$
\begin{aligned}
\mathbb{E}[X]+b \int_{\ell}^{y} p(x) d x & =\left(\frac{\ell+w}{2}\right)+b\left(\frac{y-\ell}{w-\ell}\right) \\
& =\left(\frac{b}{w-\ell}\right) y+\left(\frac{\ell+w}{2}\right)-\frac{b \ell}{w-\ell}
\end{aligned}
$$

The expected cost of the algorithm is of the form $\gamma+\beta y$ where $\gamma$ and $\beta$ are constants, independent of $y$. We know that the optimum cost is $y$. We ideally want the expected cost of the algorithm to be of the form $\beta y$ without the constant $\gamma$. This will make the competitive ratio $\beta$. It turns out that this strategy results in the optimal competitive algorithm as we show in Section 6. In order to make the constant term zero, we want to find $w$ such that

$$
\left(\frac{\ell+w}{2}\right)-\frac{b \ell}{w-\ell}=0
$$

Solving for $w$, we get

$$
w^{2}=\ell^{2}+2 b \ell \Longrightarrow w=\ell \sqrt{1+2\left(\frac{b}{\ell}\right)} .
$$

Therefore

$$
\lambda_{r} y=\frac{b}{w-\ell} y=\frac{b}{\ell\left(\sqrt{1+2\left(\frac{b}{\ell}\right)}-1\right)} y
$$

Therefore

$$
\lambda_{r}=\frac{\left(\frac{b}{\ell}\right)}{\sqrt{1+2\left(\frac{b}{\ell}\right)}-1}=\frac{1}{2}\left[1+\sqrt{1+2\left(\frac{b}{\ell}\right)}\right]
$$

where the first equality follows from the definition of $\lambda$ and the second equality follows by rationalizing the denominator, Figure 1 shows the probability density function as well as the competitive ratio for the randomized competitive algorithm. Note that the competitive ratio is $\lambda_{r}$ from $\ell$ to $w$ and then asymptotically drops to one when $y$ increases beyond $w$. Note that the competitive ratio of the deterministic algorithm is

$$
\lambda_{d}=\frac{1}{2}\left[1+\sqrt{1+4\left(\frac{b}{\ell}\right)}\right]
$$

and the expected competitive ratio of the randomized algorithm is

$$
\lambda_{r}=\frac{1}{2}\left[1+\sqrt{1+2\left(\frac{b}{\ell}\right)}\right]
$$

\subsection{Randomized Competitive Algorithm for $\alpha>0$ for Large $u$}

We now address the problem of determining a randomized competitive algorithm for the case where $\alpha>0$. As in the case for $\alpha=0$ the optimal pdf does not satisfy the principle of equality. The caterer chooses $x$ with probability density function $p(x)$. This $p(x)$ is chosen carefully to minimize the expected competitive ratio. For any given $y$, the expected cost when the allocation is done with probability density function $p(x)$ is

$$
\mathbb{E}[C(y)]=\int_{\ell}^{y}[x+\alpha(y-x)+b] p(x) d x+\int_{y}^{\infty} x p(x) d x
$$

and the optimal cost if $y$ is known ahead of time is $y$. Regrouping the $x$ terms from both the integrals, we get

$$
\mathbb{E}[C(y)]=\mathbb{E}[X]+\int_{\ell}^{y}[\alpha(y-x)+b] p(x) d x=\lambda_{r} y
$$

where $\lambda_{r}$ is the competitive ratio. Differentiating with respect to $y$ we get

$$
\alpha \int_{\ell}^{y} p(x) d x+b p(y)=\lambda_{r}
$$



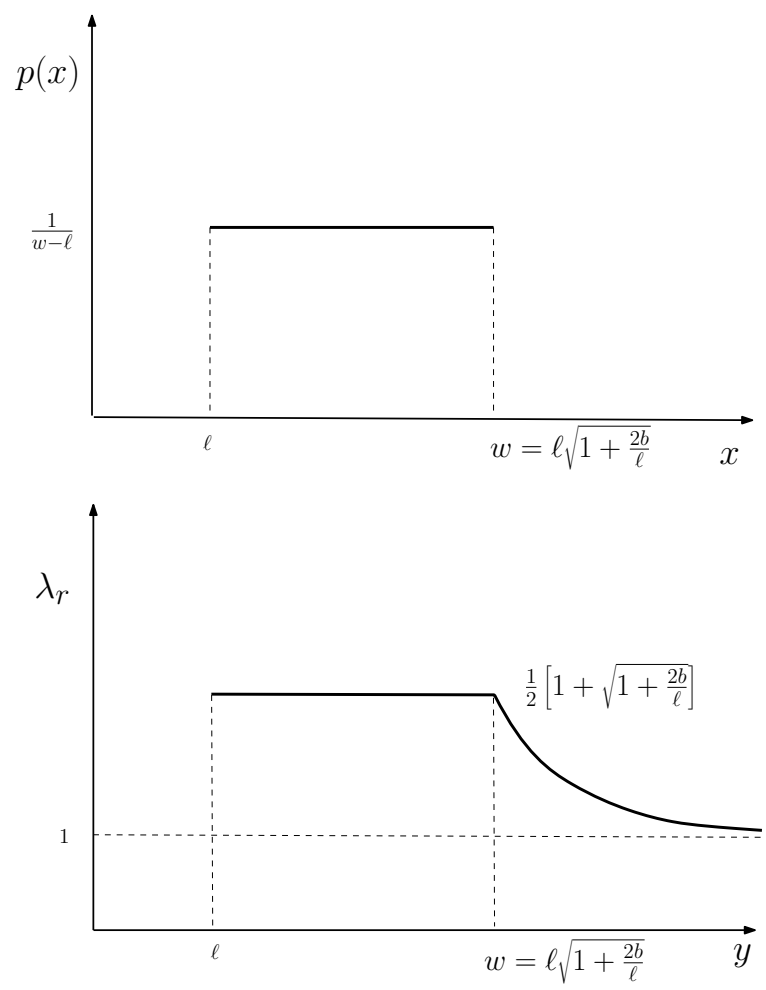

Figure 1: Probability Density Function as a function of $x$ and Competitive Ratio as a function of $y$ for the $\alpha=0$ Case

Differentiating again with respect to $y$, we get

$$
\alpha p(y)+b p^{\prime}(y)=0 .
$$

The solution to this system is

$$
p(x)=A e^{-\alpha x / b}
$$

for some constant $A$. The lower bound for this distribution is $\ell$. We let $w$ to be the upper bound. The value of $w$ will be calculated later. Therefore

$$
p(x)=k e^{-\alpha x / b} \quad \ell \leq x \leq w
$$

where

$$
k=\frac{\alpha}{b} \frac{1}{e^{-\frac{\alpha \ell}{b}}-e^{-\frac{\alpha w}{b}} .}
$$

Therefore the caterer picks the initial assignment $x$ according to the probability density function $p(x)$. We use $X$ to represent the random initial assignment. Then

$$
\mathbb{E}[X]=\frac{k b}{\alpha^{2}}\left[(\alpha \ell+b) e^{-\frac{\alpha \ell}{b}}-(\alpha w+b) e^{-\frac{\alpha w}{b}}\right]
$$

Plugging $p(x)$ in the second term of Equation [6], we get

$$
\int_{\ell}^{y}[\alpha(y-x)+b] p(x) d x=k b(y-\ell) e^{-\frac{\alpha \ell}{b}} .
$$
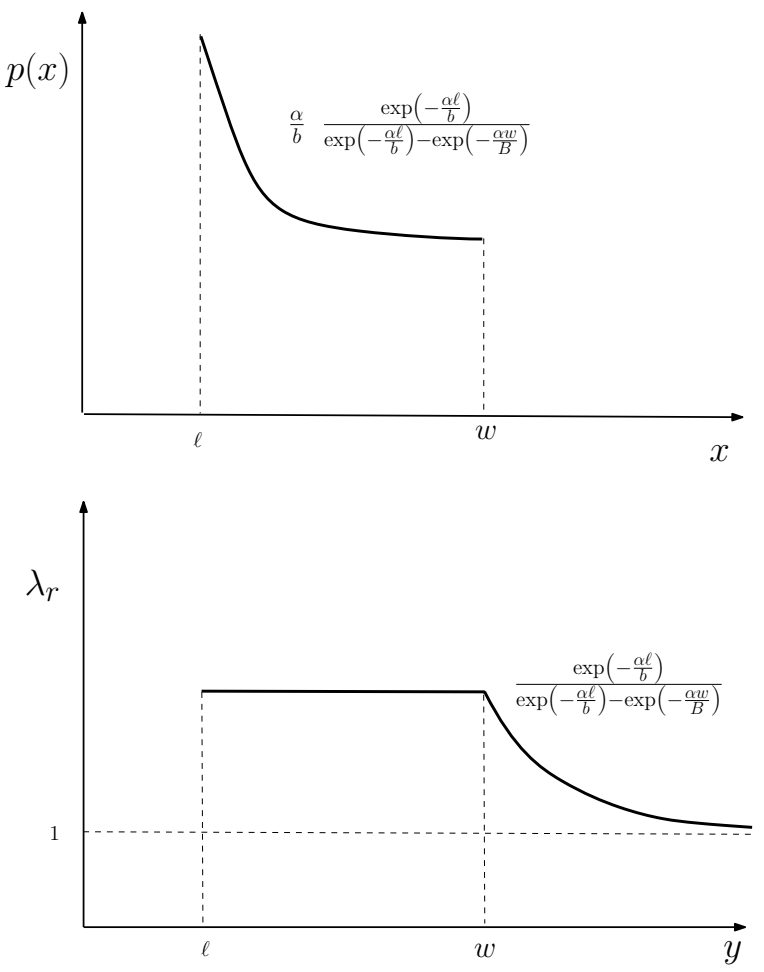

Figure 2: Probability Density Function as a function of $x$ and Competitive Ratio as a function of $y$ for the $\alpha>0$ Case

Plugging these expressions in Equation [6] and simplifying, we get

$$
\begin{aligned}
\lambda_{r} y & =\frac{k b}{\alpha^{2}}\left[\left(b+\alpha \ell-\alpha^{2} \ell\right) e^{-\frac{\alpha \ell}{b}}-(b+\alpha w) e^{-\frac{\alpha w}{b}}\right] \\
& +k b y e^{-\frac{\alpha \ell}{b}}
\end{aligned}
$$

As in the case for $\alpha=0$, we want the expected cost to be a linear function of $y$ without a constant term. In order to make the constant term zero, we want to find $w$ such that

$$
(b+\alpha w) e^{-\frac{\alpha w}{b}}=\left(b+\alpha \ell-\alpha^{2} \ell\right) e^{-\frac{\alpha \ell}{b}}
$$

This equation has a solution only if

$$
b+\alpha \ell-\alpha^{2} \ell \geq 0 .
$$

Note that this is exactly the same as Equation [4]. Therefore, the set of $\alpha$ for which the randomized algorithm provides a competitive guarantee better than $\alpha$ is also the set of $\alpha$ for which the deterministic competitive algorithm has a competitive ratio $\lambda_{r}^{1}$. Once we solve for $w$ in Equation [10] it determines the normalizing constant $k$ by equation [7]. The value 
of $w$ can be represented in terms of Lambert $W$-function as

$$
w=\frac{b}{\alpha} W\left[\frac{\alpha \ell\left(b-\alpha \ell-\alpha^{2} \ell\right)}{b^{2}}+1\right]
$$

(Lambert $W$ function is defined as follows: If $x e^{x}=z$ then $x=W(z))$. Since we have made the constant term zero, the coefficient of $y$ in Equation [9] is the competitive ratio. Therefore,

$$
\lambda_{r}=k b \exp \left(-\frac{c \ell}{b}\right)
$$

Substituting for $k$ from Equation[7],

$$
\lambda_{r}=\frac{\alpha e^{-\frac{\alpha \ell}{b}}}{e^{-\frac{\alpha \ell}{b}}-e^{-\frac{\alpha w}{b}}} .
$$

Substituting from Equation [10], we can write

$$
\lambda_{r}=\frac{b+\alpha w}{(w-\ell)+\alpha \ell}
$$

\section{Computing the value of $w$ Numerically}

It is easy to devise a iterative scheme to solve for $w$. We take log on both sides of Equation [10] and rearrange terms to get

$$
w=\ell+\frac{b}{\ell} \log \left[\frac{b+\alpha w}{b+\alpha \ell-\alpha^{2} \ell}\right] .
$$

In the iterative scheme we start off with $w_{0}=\ell$ and the value of $w$ in iteration $i$ represented by $w_{i}$ is computed as

$$
w_{i}=\ell+\frac{b}{\ell} \log \left[\frac{b+\alpha w_{i-1}}{b+\alpha \ell-\alpha^{2} \ell}\right] \quad i=1,2 \ldots
$$

We can stop when $\left|w_{i}-w_{i-1}\right|<\epsilon$.

In this section, we considered randomized competitive algorithms for the Caterer's Problem ignoring the upper bound. If the specified upper bound $u$ is greater than the computed value of $w$, then we use the probability density function derived in this section. If the value of $u<w$, then it is possible to improve the competitive ratio by taking into consideration the upper bound $u$.

\section{IMPROVING THE COMPETITIVE RATIO FOR SMALL UPPER BOUNDS}

We outline how to improve the expected competitive ratio of the randomized algorithm when the specified upper bound $u$ is less than the value of $w$ computed in the last section. In designing the shape of the optimal probability density function for the randomized algorithm, we did not use any information about the upper bound. Therefore, the shape of the probability density function is determined by the value of $\alpha$. In particular, if $\alpha=0$, the probability density function is uniform and if $\alpha>0$, the probability density function will be exponential. Since the upper bound is $u$, the algorithm will not put any mass beyond $u$. The idea then it to truncate the distribution at $u$. In the next two sections we derive the new distribution with the upper bound for the case $\alpha=0$ and $\alpha>0$.

\subsection{Competitive Algorithm for $\alpha=0$ for Small $u$}

We now consider the case when $\alpha=0$. If $u \geq w$, then the probabilistic algorithm developed in the last section is optimal. If $u<w$, then it is possible to improve the competitive ratio by changing the probability density function. In the last section the value of $w$ was chosen carefully to eliminate the constant term in the expected cost. We will use the same approach in this section. Since $u$ is given, we cannot choose the upper bound but instead we add a point mass $\phi$ at $u$ and scale down the uniform distribution between $\ell$ and $u$ by a factor of $1-\phi$. The value of $\phi$ is chosen carefully to again eliminate the constant term in the expected cost. Note that the probability density function for the initial allocation $X$ is a mixture of a scaled uniform distribution $\mathbf{U}(\ell, u)$ between $\ell$ and $u$ and a point mass $\phi$ at $u$.

$$
X \sim \begin{cases}\mathbf{U}(\ell, u) & \text { with probability } 1-\phi \\ u & \text { with probability } \phi\end{cases}
$$

Then

$$
\begin{aligned}
\mathbb{E}[X]=\int_{\ell}^{u} p(x) d x+u \alpha & =(1-\phi)\left(\frac{\ell+u}{2}\right)+u \phi \\
& =\frac{1}{2}(\ell+u)+\frac{\phi}{2}(u-\ell)
\end{aligned}
$$

The expected cost $\mathbb{E}[C(y)]$ when the number of guests is $y$ is therefore

$$
\begin{aligned}
\mathbb{E}[C(y)]= & \mathbb{E}[X]+b \int_{\ell}^{y} p(x) d x \\
= & \frac{1}{2}(\ell+u)+\frac{c \phi}{2}(u-\ell)+b(1-\phi)\left(\frac{y-\ell}{u-\ell}\right) \\
= & \frac{1}{2}(\ell+u)+\frac{\phi}{2}(u-\ell)-b(1-\alpha)\left(\frac{\ell}{u-\ell}\right) \\
& +b(1-\alpha)\left(\frac{1}{u-\ell}\right) y
\end{aligned}
$$

We want the constant term in the expected cost to be zero. Therefore,

$$
\frac{1}{2}(\ell+u)+\frac{\phi}{2}(u-\ell)-b(1-\phi)\left(\frac{\ell}{u-\ell}\right)=0 .
$$

Solving for $\phi$, we get

$$
\phi=\frac{2 b \ell-\left(u^{2}-\ell^{2}\right)}{2 b \ell+(u-\ell)^{2}}
$$

The competitive ratio $\lambda_{r}$ is the coefficient of $y$ and is given by

$$
\lambda_{r}=(1-\phi) \frac{b}{(u-\ell)} .
$$

Substituting for $\phi$, we get

$$
\lambda_{r}=\frac{2 u b}{2 b \ell+(u-\ell)^{2}} .
$$

Note that when

$$
u=\ell \sqrt{1+2 \theta}
$$


is the limiting case where the upper bound does not impact the competitive ratio and in this case $\phi=0$ and

$$
\lambda_{r}=\frac{1}{2}\left[1+\sqrt{1+2\left(\frac{b}{\ell}\right)}\right]
$$

which is the same as Equation [5].

In general, when $1 \leq \frac{u}{\ell}<\sqrt{1+2\left(\frac{b}{\ell}\right)}$, the expected competitive ratio of the modified randomized algorithm with the scaled uniform distribution will be strictly better than the randomized algorithm with just the uniform distribution. It
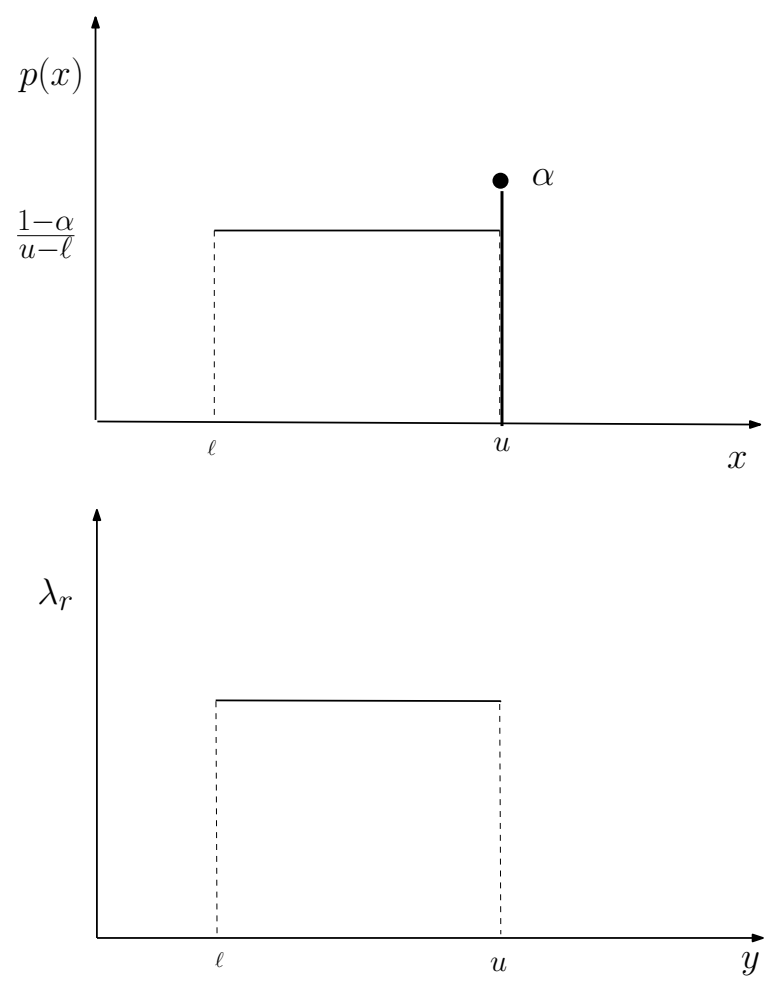

Figure 3: Probability density function and Competitive Ratio given lower bound $\ell$ and upper bound $u$.

is easy to verify that if $u$ satisfies Equation [11], (that is $u=w$ ), then the competitive ratio reduces to the same expression as Equation [12].

\subsection{Competitive Algorithm for $\alpha>0$ for Small} $u$

As in the case of $\alpha=0$, it is possible to improve the competitive ratio of the randomized algorithm when $\alpha>0$ if the upper bound $u<w$, where the value of $w$ is computed by solving Equation [10]. As in the case of $\alpha=0$, we scale the distribution (in this case the exponential distribution) and add a point mass at $u$. The scaling factor is chosen carefully to eliminate the constant term when computing the expected cost. We define

$$
X^{\prime} \sim \begin{cases}q(x)=k^{\prime} e^{-\alpha x / b} & \ell \leq x \leq u \\ u & \text { with probability } 1-\phi \\ \text { with probability } \phi\end{cases}
$$

where

$$
k^{\prime}=\frac{\alpha}{b} \frac{1}{e^{-\frac{\alpha \ell}{b}}-e^{-\frac{\alpha u}{b}}} .
$$

$$
\mathbb{E}\left[X^{\prime}\right]=\frac{k^{\prime} b(1-\phi)}{\alpha^{2}}\left[(\alpha \ell+b) e^{-\frac{\alpha \ell}{b}}-(\alpha u+b) e^{-\frac{\alpha u}{b}}\right]+\phi u
$$

Plugging $q(x)$ in the second term of Equation [6], we get

$$
\int_{\ell}^{y}[\alpha(y-x)+b] q(x) d x=k^{\prime} b(1-\phi)(y-\ell) e^{-\frac{\alpha \ell}{b}} .
$$

As in the last section, we want the constant term to go to zero so that the expected competitive ratio is a linear function of $y$ (without a constant). Setting the constant term to zero, we get

$$
\begin{aligned}
(1-\phi)\left[\left(\alpha \ell+b-\alpha^{2} \ell\right) e^{-\frac{\alpha \ell}{b}}\right. & \left.-(\alpha u+b) e^{-\frac{\alpha u}{b}}\right] \\
+ & \phi \alpha u\left(e^{-\frac{\alpha \ell}{b}}-e^{-\frac{\alpha u}{b}}\right)=0
\end{aligned}
$$

Solving for $1-\phi$, we get

$$
1-\phi=\frac{\alpha u\left(e^{-\frac{\alpha \ell}{b}}-e^{-\frac{\alpha u}{b}}\right)}{b e^{-\frac{\alpha u}{b}}-\left(\alpha \ell+b-\alpha^{2} \ell-\alpha u\right) e^{-\frac{\alpha \ell}{b}}}
$$

The expected competitive ratio is

$$
\begin{aligned}
\lambda_{r} & =(1-\phi) k^{\prime} b e^{-\frac{\alpha \ell}{b}} \\
& =\frac{\alpha^{2} u e^{-\frac{\alpha \ell}{b}}}{b e^{-\frac{\alpha u}{b}}-\left(\alpha \ell+b-\alpha^{2} \ell-\alpha u\right) e^{-\frac{\alpha \ell}{b}}}
\end{aligned}
$$

It is easy to show that when $u=w$, then the competitive ratio reduces to the same expression as Equation [12]. We have derived the expected competitive ratio of a randomized competitive algorithm for any non-negative value of $\alpha$. In the next section, we show that the competitive ratios obtained are optimal.

\section{COMPUTING LOWER BOUNDS ON THE COMPETITIVE RATIO}

We now prove that the competitive ratios obtained in the paper are tight by showing lower bounds on the competitive ratio that match the achieved competitive ratio. We outline the proofs in the case of large $u$, and the analogous proof for small $u$ is similar and omitted. We use Yao's lemma [7] to show the lower bounds. Yao's lemma, which is a restatement of the minmax theorm in game theory, shows that the best expected competitive ratio that is achievable by a randomized algorithm is lower bounded by the competitive ratio that is achieved by the best deterministic algorithm for a randomized demand. 
THEOREM 6.1. Let TC $(x, y)$ be the cost when an initial allocation $x$ is made when the demand is $y$ and OPT $(y)$ denote the optimal cost when the demand is $y$. Let $p(x)$ be a probability distribution over the initial allocation $x$, and let $q(y)$ be a probability distribution over the demand $y$. Let $X$ denote a initial allocation chosen according to $p$ and let $Y$ denote a demand chosen according to $q$. Then,

$$
\max _{y} \mathbb{E}\left[\frac{T C(X, y)}{O P T(y)}\right] \geq \min _{x} \mathbb{E}\left[\frac{T C(x, Y)}{O P T(Y)}\right] .
$$

The left hand side is the worst case expected competitive ratio and the randomization is over the initial allocation $x$. The right hand side is competitive ratio achieved by the optimal (deterministic) initial allocation when the demand $Y$ is a random variable chosen according to some probability distribution $q(y)$. The idea then is to carefully construct a probability distribution $q(y)$ over the demand $y$ and show that no deterministic algorithm can achieve a competitive ratio that is better than the competitive ratio derived in the paper.

\subsection{Lower Bound on the Competitive Ratio for $\alpha=0$}

We first consider the problem where $\alpha=0$. From Section 4.1 , we know that In this case the competitive ratio that is achieved by the randomized algorithm is $\lambda_{r}=\frac{1}{2}\left[1+\sqrt{1+2\left(\frac{b}{\ell}\right)}\right]$ and we set $w=\ell \sqrt{1+2\left(\frac{b}{\ell}\right)}$. We now give a distribution $q(y)$ over the demand $y$ such that the best deterministic algorithm achieves a competitive ratio of $\lambda_{r}$. This implies that no competitive algorithm can achieve an expected competitive ratio lower than $\lambda_{r}$. The strategy that we use is to find a demand distribution $q(y)$ such that the competitive ratio is independent of the initial allocation $x$. This turns out to be the distribution that gives a lower bound of $\lambda_{r}$ on the competitive ratio.

THEOREM 6.2. Consider a distribution $q(y)$ over the demand y comprising of a point mass at $\ell$ and a continuous density between $\ell$ and $w=\ell \sqrt{1+2\left(\frac{b}{\ell}\right)}$ given by

$$
q(y)= \begin{cases}\frac{\ell(\ell+b-w)}{w(w-\ell)} & \text { if } y=\ell \\ \frac{1}{w(w-\ell)} y & \text { if } \ell<y \leq w .\end{cases}
$$

The best competitive ratio that can be achieved by any deterministic algorithm is $\lambda_{r}$.

Proof. We first verify that the given probability density function is valid by ensuring that it is non-negative and sums to one. In order to show that the pdf is non-negative, we have to verify that $\ell+b \geq w$ so that the point mass is non-negative. This condition is equivalent to verifying that $1+\frac{b}{\ell} \geq \frac{w}{\ell}$. From the definition of $w$, note that $\frac{w}{\ell}=\sqrt{1+\frac{2 b}{\ell}}$. Therefore, we want to verify that $1+\frac{b}{\ell} \geq \sqrt{1+\frac{2 b}{\ell}}$. Squaring both sides can cancelling out the common terms, we get $\left(\frac{b}{\ell}\right)^{2} \geq 0$ which is true. It is also easy to show that

$$
\frac{\ell(\ell+b-w)}{w(w-\ell)}+\int_{\ell}^{w} \frac{1}{w(w-\ell)} y d y=1
$$

using the fact that $\ell^{2}=w^{2}-2 b \ell$. We now compute the competitive ratio of any deterministic algorithm that makes an initial allocation of $x$. Note that the optimal solution when the demand is $y$ is $y$. Therefore, the expected competitive ratio for a fixed value of $x$ is

$$
\begin{aligned}
& \frac{T C(x, Y)}{O P T(Y)}=\frac{T C(x, Y)}{Y}= \begin{cases}\frac{x}{\ell} & \text { with prob } \frac{\ell(\ell+b-w)}{w(w-\ell)} \\
\frac{x}{y} & \text { with prob } \frac{y}{w(w-\ell)} \text { if } \ell<y \leq w\end{cases} \\
& \text { The expected competitive ratio } \mathbb{E}_{y}\left[\frac{T C(x, Y)}{Y}\right] \\
& \begin{aligned}
\mathbb{E}_{y}\left[\frac{T C(x, Y)}{Y}\right] & =\frac{\ell(\ell+b-w)}{w(w-\ell)} \frac{x}{\ell}+x \int_{\ell}^{x} \frac{1}{w(w-\ell)} d y+(x+b) \int_{a}^{w} \frac{1}{w(w-\ell)} d y \\
& =\frac{b}{w-\ell}=\frac{b}{\ell \sqrt{1+2\left(\frac{b}{\ell}\right)}-\ell}=\frac{1}{2}\left[1+\sqrt{1+2\left(\frac{b}{\ell}\right)}\right]
\end{aligned}
\end{aligned}
$$

Note that the last expression is independent of $x$ which is the same as Equation [5].

Therefore, we have shown that the lower bound on the competitive ratio matches the achieved expected competitive ratio and the expected competitive ratio is optimal. We

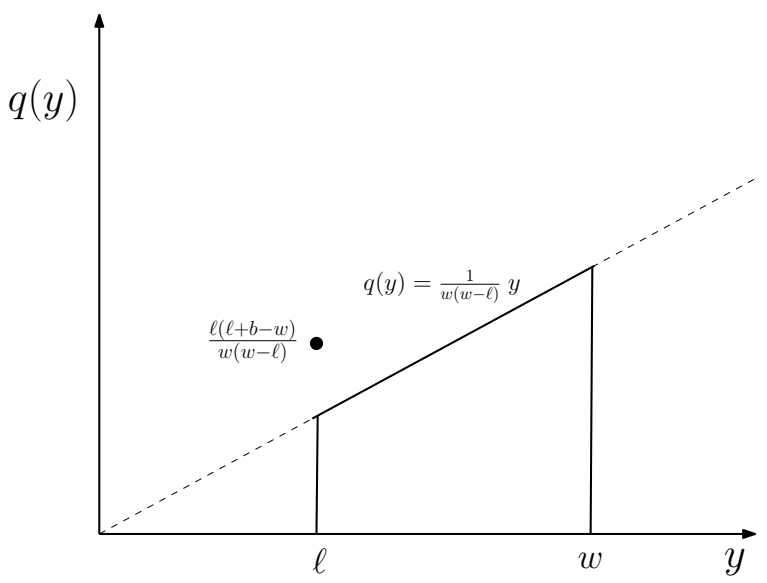

Figure 4: Probability Distribution of the Worst Case Demand for $\alpha=0$. Note the point mass at $y=\ell$.

now derive the lower bound on the competitive ratio for the case of $\alpha>0$.

\subsection{Lower Bound for $\alpha>0$}

As in the case of $\alpha=0$, we choose the distribution $q(y)$ such that the expected competitive ratio is independent of the initial allocation $x$. The distribution has a point mass at $\ell$ and a continuous density function between $\ell$ and $w$. Assume that the point mass at $\ell$ is $\theta$ and the density function is

$$
q(y)=k(1-\theta) y e^{\alpha y / b} \quad \ell \leq y \leq w
$$


where $k$ is a constant chosen to ensure that the probability distribution is normalized. We visualize this distribution in Figure 5. By direct integration, it is easy to show that

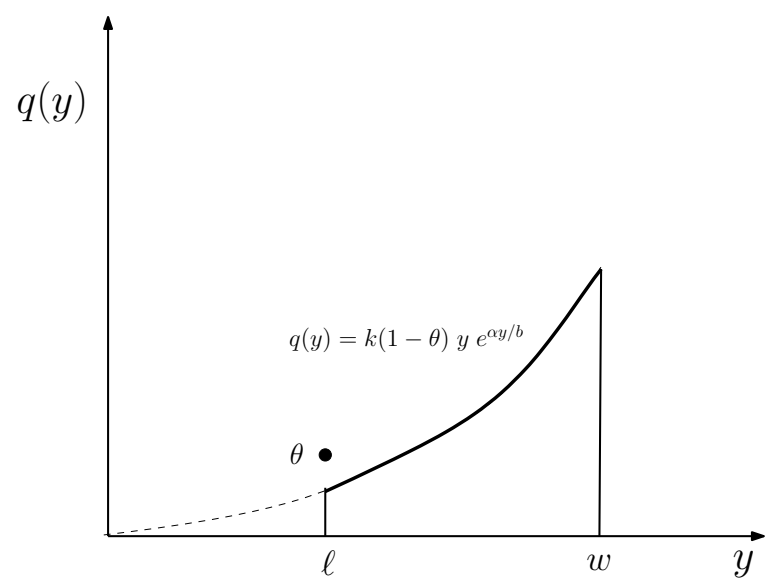

Figure 5: Probability Distribution of the Worst Case Demand for general $\alpha$.

$$
k^{-1}=\frac{b}{\alpha^{2}}\left[(b-\alpha \ell) e^{\alpha \ell / b}+(\alpha w-b) e^{\alpha w / b}\right] .
$$

If the initial allocation is $a$ then the expected competitive ratio is

$$
\begin{aligned}
\mathbb{E}_{y}\left[\frac{T C(x, Y)}{Y}\right]= & \theta \frac{x}{\ell}+x \int_{\ell}^{x} \frac{q(y)}{y} d y \\
& +\int_{x}^{w}[x+b+\alpha(y-x)] \frac{q(y)}{y} d y \\
= & \theta \frac{x}{\ell}+x b k(1-\theta)\left(\frac{e^{\alpha w / b}}{\alpha}-\frac{e^{\alpha \ell / b}}{\alpha}-e^{\alpha w / b}\right) \\
& +b k w(1-\theta) e^{\alpha w / b}
\end{aligned}
$$

We choose $\theta$ to make the expression independent of the value of the initial assignment $x$ by setting

$$
\frac{\theta}{\ell}=b k(1-\theta)\left[\left(1-\frac{1}{\alpha}\right) e^{\alpha w / b}+\frac{1}{\alpha} e^{\alpha \ell / b}\right] .
$$

Solving for $\theta$, we get

$$
\theta=\frac{b k \ell M}{1+b k \ell M}
$$

where

$$
M=\left[\left(1-\frac{1}{\alpha}\right) e^{\alpha w / b}+\frac{1}{\alpha} e^{\alpha \ell / b}\right] .
$$

The expected competitive ratio is then

$$
\mathbb{E}_{y}\left[\frac{T C(x, Y)}{Y}\right]=b k w(1-\theta) e^{\alpha w / b} .
$$

Substituting the value of $\theta$, we get

$$
\begin{aligned}
\mathbb{E}_{y}\left[\frac{T C(x, Y)}{Y}\right] & =\frac{k b w e^{\alpha w / b}}{1+k b \ell M} \\
& =\frac{b+\alpha w}{w-\ell+\alpha \ell}=\lambda_{r}
\end{aligned}
$$

Note that the lower bound on the competitive ratio matches the expected competitive ratio shown in Equation [12]. Therefore, the competitive ratio of the randomized algorithms derived in Section 4 are optimal. The techniques used in this section, can be used to show that the randomized competitive algorithms derived in Section 5 for the small upper bound case are also optimal.

\subsection{Conclusion}

In this paper, we considered a resource allocation problem to meet a demand about which we only have minimal information. The motivation for studying this problem is resource allocation for controlling pandemics and for reserving resources in the cloud for new applications. We derived optimal deterministic and randomized algorithms for this problem. The problem that we addressed in this paper is a single stage resource allocation problem where the allocation decision is made once. We are working on extending the algorithm to multi-stage resource allocation problems where the demand is revealed over multiple stages.

\section{REFERENCES}

[1] Francis Y Edgeworth. 1888. The mathematical theory of banking. Journal of the Royal Statistical Society, 51, 1, 113-127.

[2] Evan L Porteus. 2008. The newsvendor problem. In Building Intuition. Springer, 115-134.

[3] Yan Qin, Ruoxuan Wang, Asoo J Vakharia, Yuwen Chen, and Michelle MH Seref. 2011. The newsvendor problem: review and directions for future research. European Journal of Operational Research, 213, 2, 361374.

[4] Tsan-Ming Choi. 2012. Handbook of Newsvendor problems: Models, extensions and applications. Volume 176. Springer Science \& Business Media.

[5] Helena Gaspars-Wieloch. 2017. Newsvendor problem under complete uncertainty: a case of innovative products. Central European Journal of Operations Research, 25, 3, 561-585.

[6] Peijun Guo and Xiuyan Ma. 2014. Newsvendor models for innovative products with one-shot decision theory. European Journal of Operational Research, 239, 2, 523-536.

[7] Allan Borodin and Ran El-Yaniv. 2005. Online computation and competitive analysis. cambridge university press.

[8] Anna R Karlin, Mark S Manasse, Larry Rudolph, and Daniel D Sleator. 1988. Competitive snoopy caching. Algorithmica, 3, 1-4, 79-119.

[9] Steven S Seiden. 2000. A guessing game and randomized online algorithms. In Proceedings of the thirty-second annual ACM symposium on Theory of computing, 592-601.

[10] Andrew Chi-Chin Yao. 1977. Probabilistic computations: toward a unified measure of complexity. In 18th Annual Symposium on Foundations of Computer Science (sfcs 1977). IEEE, 222-227. 